\title{
Power Dependent Noise Performance of fs Supercontinuum Generation in Normal Dispersion Fibers with a Long Zero-dispersion Wavelength
}

\author{
Shreesha Rao D. S. ${ }^{1}$, Rasmus D. Engelsholm ${ }^{1}$, Iván B. Gonzalo ${ }^{1}$, Binbin Zhou ${ }^{1}$, Patrick Bowen ${ }^{2}$, \\ Peter M. Moselund ${ }^{2}$, Morten Bache ${ }^{1}$, and Ole Bang ${ }^{1,2}$ \\ 1. DTU Fotonik, Department of Photonics Engineering, Technical University of Denmark, Ørsteds Plads, 2800 Kongens Lyngby, Denmark. \\ 2. NKT Photonics A/S, Blokken 84, 3460 Birkerød, Denmark.
}

One of the requirements for generating a low noise supercontinuum (SC) in a normal dispersion fiber using a femtosecond (fs) pump is to have the minimum absolute dispersion wavelength to be close to zero in order to avoid parametric Raman noise [1]. SC generation in all-normal dispersion fibers can under certain requirements provide a highly coherent spectrum [2,3], which is of key importance to a large range of imaging and spectroscopy applications. Key requirements to avoid Raman noise and noise from polarization mode instability (PMI) is to have a sufficiently low peak power $\left(\mathrm{P}_{0}\right)$, short pulse length $\left(\mathrm{T}_{0}\right)$, short fiber length, and high fiber birefringence [4].

Silica microstructured fibers (MSFs) pumped with $1550 \mathrm{~nm}$ fs lasers is an attractive platform to generate a low noise SC due to their technological maturity. We therefore fabricated a low-loss silica MSF (using 2 cladding sections with different hole sizes) with weak dispersion at $1500 \mathrm{~nm}$ and experimentally studied SC generation at low peak powers using a $1550 \mathrm{~nm}, 125 \mathrm{fs}$ pump [5]. The group velocity dispersion $\left(\beta_{2}\right)$ profile of the fiber is shown in Fig. 1(a). Since the dispersion at the pump is weak, a low $\mathrm{P}_{0}$ can be used to generate a broad SC. A further advantage of using low peak powers is the possibility of avoiding PMI. The requirement of weak dispersion at $1550 \mathrm{~nm}$ leads to a fiber design with a zero dispersion wavelength (ZDW) at $1.8 \mu \mathrm{m}$. The SC generated with up to $\mathrm{P}_{0}=9 \mathrm{~kW}$ does not cross the ZDW and has low relative intensity noise (RIN) as was demonstrated numerically and experimentally [see Fig. 1(b)].
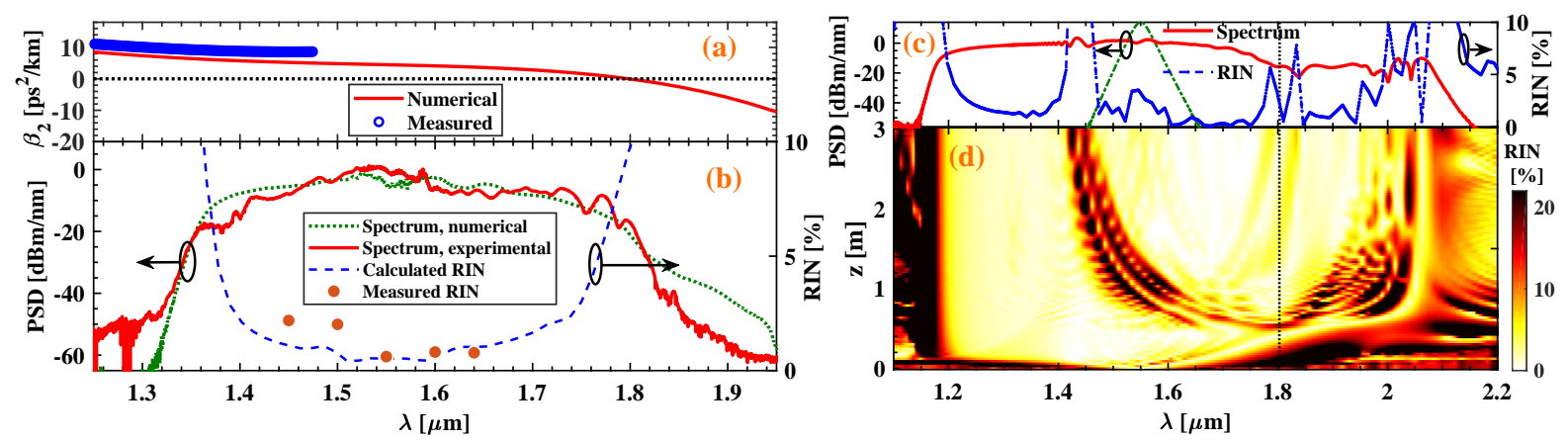

Fig. 1. (a) Numerically calculated (red) dispersion and the dispersion measured experimentally (blue) using a white light interferometry set up. (b) Numerically calculated spectrum (green) and RIN (blue) after $\mathrm{z}=3 \mathrm{~m}$ and $\mathrm{P}_{0}=9 \mathrm{~kW}$ and the experimentally measured spectrum (red) and the RIN (dots) measured using $12 \mathrm{~nm}$ bandwidth bandpass filters. (c) Numerically calculated spectrum (red) and RIN (blue) after $\mathrm{z}=3 \mathrm{~m}$ and $\mathrm{P}_{0}=45 \mathrm{~kW}$. (d) Evolution of RIN along $3 \mathrm{~m}$ of the fiber with $\mathrm{P}_{0}=45 \mathrm{~kW}$.

To study the effect of higher input power, where the SC crosses into the anomalous dispersion region (ADR), we performed numerical modelling, taking into account both quantum noise and laser amplitude noise by adding $1 \%$ fluctuation in $\mathrm{P}_{0}$ with constant $\mathrm{P}_{0} \mathrm{~T}_{0}[4,6]$. At $\mathrm{P}_{0}=45 \mathrm{~kW}$ the broadening is initially from self-phase modulation and optical wave breaking in the normal dispersion region (NDR), but later the SC broadens into the ADR and develops into solitons. These solitons generate trapped dispersive waves that are very noisy and move through the low-noise spectrum in the NDR [see Fig. 1(c+d)]. Our results using the novel MSF for low-noise normal dispersion SC generation at $1550 \mathrm{~nm}$, demonstrate that a long wavelength ZDW can be tolerated, as long as the peak power is sufficiently low to avoid power crossing into the ADR.

Funding. European Union's Horizon 2020 research and innovation program under the Marie Curie grant agreement No. 722380 (SUPUVIR).

\section{References}

[1] A. M. Heidt, J. S. Feehan, J. H. V. Price, and T. Feurer. "Limits of coherent supercontinuum generation in normal dispersion fibers." J. Opt. Soc. Am. B, 34, 764-775 (2017).

[2] A. M. Heidt, A. Hartung, G. W. Bosman, P. Krok, E. G. Rohwer, H. Schwoerer, and H. Bartelt. "Coherent octave spanning near-infrared and visible supercontinuum generation in all-normal dispersion photonic crystal fibers." Opt. Exp., 19, 3775-3787 (2011).

[3] M. Klimczak, B. Siwicki, P. Skibiński, D. Pysz, R. Sętepień, A. Heidt, C. Radzewicz, and R. Buczyński. "Coherent supercontinuum generation up to $2.3 \mu \mathrm{m}$ in all-solid soft-glass photonic crystal fibers with flat all-normal dispersion." Opt. Exp., 22, 18824-18832 (2014).

[4] I. B. Gonzalo, R. D. Engelsholm, M. P. Sørensen, and O. Bang. "Polarization noise places severe constraints on coherence of all-normal dispersion femtosecond supercontinuum generation." Sci. Rep., 8, 6579 (2018).

[5] S. Rao D. S., R. D. Engelsholm, I. B. Gonzalo, B. Zhou, P. Bowen, P. M. Moselund, O. Bang, and M. Bache. "Ultra-low Noise Supercontinuum Generation with Flat Near-zero Normal Dispersion Fiber." arXiv preprint arXiv:1812.03877 (2018).

[6] E. Genier, P. Bowen, T. Sylvestre, J. Dudley, P. Moselund, and O. Bang. "Amplitude noise and coherence degradation of femtosecond supercontinuum generation in all-normal-dispersion fibers." J. Opt. Soc. Am. B, 36 (2019). 